\title{
Improving the Feedback Process in Medical Education
}

\author{
Shrivastava, S.R. ${ }^{1}$ \& Shrivastava, P.S. ${ }^{2}$
}

The concept of feedback in Medical Education, regardless of whether it is traditional or competence-driven, is of immense value and plays a remarkable role in the professional development of the student as well as the teacher (Shrivastava et al., 2014; Shah et al., 2016). Feedback helps the learner to bridge their existing gaps and move forward towards what is eventually intended out of them (Shrivastava et al., 2014). Even though, feedback has been linked with numerous merits, it has a negative side as well, and can account for a potential negative impact on the receivers (Hardavella et al., 2017; Wang \& Kogan, 2018). In order to avert the negative consequences of feedback, all stakeholders should understand that it should be used with a single goal of improving the performance of the learner (Shrivastava et al., 2014; Shah et al., 2016; Hardavella et al., 2017). It should be constructive, linked with a specific behavior and should not comment about general performance (Shah et al., 2016). In-fact, both the teacher and student should be considered as a team working together to achieve a common goal, and thus utmost care should be taken to maintain their relationship for the eventual success (Shah et al., 2016; Hardavella et al., 2017). Moreover, the feedback should be given at a mutually agreed time and place (Shrivastava et al., 2014).

${ }^{1}$ Medical Education Unit and Institute Research Council, Department of Community Medicine, Shri Sathya Sai Medical College \& Research Institute, Sri Balaji Vidyapeeth - Deemed to be University, Ammapettai, Nellikuppam, Chengalpet District, Tamil Nadu, India.

${ }^{2}$ Department of Community Medicine, Shri Sathya Sai Medical College \& Research Institute, Sri Balaji Vidyapeeth - Deemed to be University, Ammapettai, Nellikuppam, Chengalpet District, Tamil Nadu, India.

Corresponding Author: Dr. Saurabh RamBihariLal Shrivastava

Professor, Department of Community Medicine, Shri Sathya Sai Medical College and Research Institute, Sri Balaji Vidyapeeth (SBV) - Deemed to be University, Tiruporur - Guduvancherry Main Road, Ammapettai, Nellikuppam, Chengalpet District, Tamil Nadu - 603108, India.

Email:drshrishri2008@gmail.com
The feedback session should start with a positive aspect and subsequently address all the other relevant aspects of demonstrated skills (Shrivastava et al., 2014; Shah et al., 2016; Hardavella et al., 2017).

From the provider perspective, training is needed in giving both positive and negative feedback and thus should be aware of the goals/ learning outcomes before giving feedback (Hardavella et al., 2017; Wang \& Kogan, 2018). The aim should be to identify the root cause of the problem and so adequate preplanning and documents should be collected (Wang \& Kogan, 2018). The provider should be descriptive, non-judgmental and give their comments based on direct observations \& not on what others might comment about a specific student (Hardavella et al., 2017). The provider should listen carefully to the problems of the learner and repeat the entire process periodically (Hardavella et al., 2017; Wang \& Kogan, 2018; Alrebish, 2018).

The provider should ideally create such an environment that the learners identify their negative aspects on their own and thus they themselves take the onus for their improvement (Wang \& Kogan, 2018; Alrebish, 2018). Moreover, negative feedback which directly attacks the student should be avoided and if required, suitable examples can be used (Wang \& Kogan, 2018). Aditionally, the faculty should never accumulate the negative aspects of the learner, as a change in behaviour can be more easily accomplished when negative feedback is administered in small doses (Hardavella et al., 2017; Wang \& Kogan, 2018; Alrebish, 2018). The provider should never compare performance of one student with another (Hardavella et al., 2017).

From the receiver perspective, they have to be receptive and should have a positive attitude towards receiving feedback (Hardavella et al., 2017; Wang \& Kogan, 2018). In addition, the learners should be given a chance to give an appropriate explanation for their performance

DOI: http://doi.org/10.4038/seajme.v14i1.238

(C) SEAJME. This is an Open Access article distributed under the terms of the Creative Commons Attribution
License (http://creativecommons.org/licenses/by/4.0/), which permits unrestricted use, distribution, and
reproduction in any medium, provided the original author and source are credited


(Hardavella et al., 2017; Alrebish, 2018). The best thing will be to earmark one hour in a month's time for the mentor-mentee system and during this time students should be given freedom to discuss their personal or academic problems with their mentors. In-fact, even a drop-box can be started, in which students can put their anonymous feedback (pertaining to structural, curricular or faculty aspects), and based on the received suggestions, appropriate measures should be taken to address the same.

In conclusion, feedback is a wonderful tool in the delivery of Medical Education, provided both teacher and student work together as a team and in which learners' interests are safeguarded.

\section{References}

Alrebish, S.A. (2018) Barriers to Effective Feedback in Undergraduate Medical Education: Case study from Saudi Arabia. International Journal of Health Sciences, 12, 2, pp.31.
Hardavella, G., Aamli-Gaagnat, A., Saad, N., Rousalova, I. \& Sreter, K.B. (2017) How to Give and Receive Feedback Effectively. Breathe, 13, 4, pp.327-333.

Shah, N., Desai, C., Jorwekar, G., Badyal, D. \& Singh, T. (2016) Competency-Based Medical Education: An Overview and Application in Pharmacology. Indian Journal of Pharmacology. 48, Suppl 1, pp. S5-S9.

Shrivastava, S.R., Shrivastava, P.S. \& Ramasamy, J. (2014) Effective Feedback: An Indispensable Tool for Improvement in Quality of Medical Education. Journal of Pedagogic Development.4, 1, pp.12-20.

Wang, F.Y. \& Kogan, J.R. (2018) Concrete Steps for Empowering Learners to Create a Feedback Environment. Academic Medicine, 93, 7, pp.960. 